ANNALES

POLONICI MATHEMATICI

$86.3(2005)$

\title{
Continuity of plurisubharmonic envelopes
}

\author{
by Nihat Gokhan Gogus (Syracuse, NY)
}

\begin{abstract}
Let $D$ be a domain in $\mathbb{C}^{n}$. The plurisubharmonic envelope of a function $\varphi \in C(\bar{D})$ is the supremum of all plurisubharmonic functions which are not greater than $\varphi$ on $D$. A bounded domain $D$ is called $c$-regular if the envelope of every function $\varphi \in C(\bar{D})$ is continuous on $D$ and extends continuously to $\bar{D}$. The purpose of this paper is to give a complete characterization of $c$-regular domains in terms of Jensen measures.
\end{abstract}

1. Introduction. The plurisubharmonic envelopes of functions have been quite useful and found a lot of applications in pluripotential theory. In this paper we address the problem of their continuity. To characterize domains where continuous functions have continuous envelopes we use the notion of Jensen measures. These measures have recently attracted the attention of quite a number of mathematicians (see [CCW], [W], [CR], [R] and [P3]) and have been used to explain different phenomena of pluripotential theory.

In particular, in [W] Wikström uses them to classify domains where the Dirichlet problem for the homogeneous Monge-Ampère equation always has a continuous solution. It should be noted that different classes of plurisubharmonic functions generate different Jensen measures. The results in [W] are obtained by comparing these classes of measures. In $\S 2$ we introduce additional classes of Jensen measures which we consider as multifunctions in $C^{*}(\bar{D})$. As it happens the continuity of envelopes is a consequence of such geometric properties of these multifunctions as upper and lower semicontinuity. In $\S 3$ we establish these properties for our classes and after that in $\S 4$ we prove the main theorem. Roughly speaking, envelopes of continuous functions are continuous if and only if any limit point of Jensen measures with respect to bounded plurisubharmonic functions can be obtained as a limit of such Jensen measures from every direction. We call such domains c-regular.

2000 Mathematics Subject Classification: Primary 32U15.

Key words and phrases: plurisubharmonic envelopes, Jensen measures, pluripotential theory. 
In $\S 5$ we investigate the continuity of the Perron-Bremermann envelopes for continuous functions on the boundary. We prove for a particular class of domains that the continuity of the Perron-Bremermann envelopes is necessary and sufficient for the continuity of the plurisubharmonic envelopes.

In $\S 6$ we characterize strongly regular points, which are local plurisubharmonic peak functions, in terms of Jensen measures and prove that their set is always closed in the boundary when the domain is $c$-regular. In $\S 7$ we give an example of a smooth, strongly hyperconvex star-shaped domain where continuous functions need not have envelopes that extend continuously to the boundary. Note that by $[\mathrm{W}]$ they are continuous inside. In this example strongly regular points are not closed in the boundary.

I would like to thank Prof. Evgeny Poletsky, who suggested this problem to me, for helpful discussions.

Now let us start by introducing the classes of Jensen measures and give some basic properties.

2. Classes of Jensen measures. If $f$ is a holomorphic mapping of a neighborhood $V$ of the closure $\bar{U}$ of the unit disk $U \subset \mathbb{C}$ into an open set $D \subset \mathbb{C}^{n}$, then the measure

$$
\mu_{f}(E)=\frac{1}{2 \pi} l\left(f^{-1}(E) \cap T\right),
$$

where $T=\partial U$ and $l(A)$ is the length of a set $A \subset T$, is Jensen with barycenter $z_{0}=f(0)$. For $z \in D$ let $\mathcal{H}_{z}=\mathcal{H}_{z}(D)$ be the set of all measures $\mu_{f}$ such that $f(0)=z$.

We will frequently use a theorem proved in [P2].

THEOREM 2.1. If $\varphi$ is an upper semicontinuous function on an open set $D \subset \mathbb{C}^{n}$, then the function

$$
S \varphi(z)=\sup \{u(z): u \leq \varphi \text { is plurisubharmonic on } D\}
$$

is plurisubharmonic and equal to

$$
E \varphi(z)=\inf \left\{\int \varphi d \mu: \mu \in \mathcal{H}_{z}\right\}
$$

the plurisubharmonic envelope of $\varphi$.

Let us introduce the following classes of Jensen measures. In the following definitions $\varphi$ is a continuous function on $\bar{D}$ and $\mathcal{M}(\bar{D})$ is the class of all regular Borel measures $\mu$ on $\bar{D}$ such that $\mu(\bar{D})=1$. The set $\mathcal{M}(\bar{D})$ belongs to the dual $C^{*}(\bar{D})$ of $C(\bar{D})$ and we will endow it with the weak-* topology defined by this duality.

(i) Given $z \in D$, we denote by $\mathcal{J}_{z}=\mathcal{J}_{z}(D)$ the family of all measures $\mu \in \mathcal{M}(\bar{D})$ with compact support in $D$ such that

$$
u(z) \leq \int u d \mu
$$


for every $u$ in the set $\operatorname{PSH}(D)$ of all plurisubharmonic functions on $D$. We define the set $\overline{\mathcal{J}}_{z}$ as the weak-* closure of $\mathcal{J}_{z}$ with respect to $\mathcal{M}(\bar{D})$.

(ii) For $z \in \bar{D}$ let

$$
\widehat{\mathcal{J}}_{z}=\widehat{\mathcal{J}}_{z}(D)=\left\{\mu: \mu=\lim \mu_{j}, \mu_{j} \in \overline{\mathcal{J}}_{z_{j}}, z_{j} \rightarrow z, z_{j} \in D\right\},
$$

where the limit above should be understood as the weak-* limit.

We define the envelope

$$
\widehat{E} \varphi(z)=\widehat{E}_{D} \varphi(z)=\inf \left\{\int \varphi d \mu: \mu \in \widehat{\mathcal{J}}_{z}\right\} .
$$

(iii) If $\psi$ is a bounded function on $D$ we define its upper semicontinuous regularization $\psi^{*}$ on $\bar{D}$ as

$$
\psi^{*}(z)=\limsup _{w \rightarrow z, w \in D} \psi(w) .
$$

Let $\mathcal{J}_{z}^{\mathrm{b}}=\mathcal{J}_{z}^{\mathrm{b}}(D)(z \in \bar{D})$ be the set of all measures $\mu$ in $\mathcal{M}(\bar{D})$ such that

$$
u^{*}(z) \leq \int u^{*} d \mu
$$

for all $u$ in the set $\operatorname{PSH}^{\mathrm{b}}(D)$ of all bounded plurisubharmonic functions on $D$. Let

$$
E^{\mathrm{b}} \varphi(z)=E_{D}^{\mathrm{b}} \varphi(z)=\inf \left\{\int \varphi d \mu: \mu \in \mathcal{J}_{z}^{\mathrm{b}}\right\} .
$$

This class of Jensen measures was studied in [W].

(iv) If $z \in \bar{D}$ is a point, let $\operatorname{PSH}^{z}(D)$ be the set of all upper bounded plurisubharmonic functions on $D$ for which $\lim _{w \rightarrow z, w \in D} u(w)$ exists. We define $\mathcal{J}_{z}^{l}=\mathcal{J}_{z}^{l}(D)$ to be the set of all $\mu \in \mathcal{M}(\bar{D})$ such that

$$
u(z) \leq \int u^{*} d \mu
$$

for every $u \in \mathrm{PSH}^{z}(D)$.

(v) Given $z \in \bar{D}$, we write $\mathcal{J}_{z}^{\mathrm{c}}=\mathcal{J}_{z}^{\mathrm{c}}(D)$ for the set of all measures $\mu \in \mathcal{M}(\bar{D})$ such that

$$
u(z) \leq \int u d \mu
$$

for every $u \in \operatorname{PSH}^{\mathrm{c}}(D)=\operatorname{PSH}(D) \cap C(\bar{D})$. The class $\mathcal{J}_{z}^{\mathrm{c}}$ was introduced in $[\mathrm{CCW}]$.

Here we would like to state a few basic facts about these measures that will be used in the paper.

All Jensen measures are necessarily probability measures since the constant functions 1 and -1 are pluriharmonic everywhere. Note that the class $\widehat{\mathcal{J}}_{z}$ need not be convex, but the classes $\overline{\mathcal{J}}_{z}, \mathcal{J}_{z}^{\mathrm{b}}, \mathcal{J}_{z}^{l}$ and $\mathcal{J}_{z}^{\mathrm{c}}$ are convex. The compactness of $\overline{\mathcal{J}}_{z}, \widehat{\mathcal{J}}_{z}$ and $\mathcal{J}_{z}^{\mathrm{c}}$ is obvious. Let us show that $\mathcal{J}_{z}^{\mathrm{b}}$ is compact. First we need the following lemma: 
Lemma 2.2. Let $s$ be an upper semicontinuous function on $\bar{D}$ and $\left\{\mu_{j}\right\} \subset$ $C^{*}(\bar{D})$ be a sequence of measures converging weak-* to a measure $\mu \in C^{*}(\bar{D})$. Then

$$
\limsup _{j} \int s d \mu_{j} \leq \int s d \mu .
$$

Proof. There exist functions $\varphi_{k} \in C(\bar{D})$ so that $\varphi_{k} \downarrow s$ on $\bar{D}$. Then

$$
\limsup _{j} \int s d \mu_{j} \leq \limsup _{j} \int \varphi_{k} d \mu_{j}=\int \varphi_{k} d \mu
$$

for all $k$. Finally, by the monotone convergence theorem,

$$
\limsup _{j} \int s d \mu_{j} \leq \int s d \mu
$$

Suppose $\mu_{j}$ is a sequence in $\mathcal{J}_{z}^{\mathrm{b}}$ that converges weak-* to $\mu \in \mathcal{M}(\bar{D})$ and take any upper bounded plurisubharmonic function $u$ on $D$. Then by Lemma 2.2,

$$
u^{*}(z) \leq \limsup _{j} \int u^{*} d \mu_{j} \leq \int u^{*} d \mu .
$$

Therefore, $\mu \in \mathcal{J}_{z}^{\mathrm{b}}$ and $\mathcal{J}_{z}^{\mathrm{b}}$ is compact. One can argue the same way to prove that $\mathcal{J}_{z}^{l}$ is compact.

We will frequently use a corollary of the Hahn-Banach theorem, so it is worth noting it here ([C, Theorem 3.9]).

Theorem 2.3. Let $X$ be a real locally convex space and $A$ and $B$ be two disjoint closed convex subsets of $X$. If $B$ is compact, then there exist a continuous linear functional $f$ on $X$ and a number $\alpha$ so that

$$
\sup \{f(b): b \in B\}<\alpha<\inf \{f(a): a \in A\} .
$$

All classes of Jensen measures are related as follows:

Lemma 2.4. If $z \in D$, then $\mathcal{H}_{z} \subset \mathcal{J}_{z} \subset \overline{\mathcal{J}}_{z}=\overline{\mathcal{H}}_{z}=\mathcal{J}_{z}^{\mathrm{b}} \subset \widehat{\mathcal{J}}_{z}$. If $z \in \bar{D}$, we have $\widehat{\mathcal{J}}_{z} \subset \mathcal{J}_{z}^{l} \subset \mathcal{J}_{z}^{\mathrm{c}}$.

Proof. The first and second inclusions follow immediately from the definitions. By a theorem of $\mathrm{Bu}$ and Schachermayer (see [BS]), $\mathcal{J}_{z} \subset \overline{\mathcal{H}}_{z}$. Since also $\mathcal{H}_{z} \subset \mathcal{J}_{z}$, we get $\overline{\mathcal{J}}_{z}=\overline{\mathcal{H}}_{z}$.

Clearly $\mathcal{J}_{z} \subset \mathcal{J}_{z}^{\mathrm{b}}$, hence $\overline{\mathcal{J}}_{z} \subset \mathcal{J}_{z}^{\mathrm{b}}$ since $\mathcal{J}_{z}^{\mathrm{b}}$ is closed. Conversely, if there exists $\mu \in \mathcal{J}_{z}^{\text {b }} \backslash \overline{\mathcal{J}}_{z}$, then by Theorem 2.3 there exists $\varphi \in C(\bar{D})$ such that

$$
\int \varphi d \mu<\inf _{\nu \in \overline{\mathcal{J}}_{z}} \int \varphi d \nu=u(z),
$$

where $u=E \varphi$ is the plurisubharmonic envelope of $\varphi$. Then $u \in \operatorname{PSH}^{\mathrm{b}}(D)$ by Theorem 2.1 and $u \leq \varphi$, but we have

$$
\int \varphi d \mu<u(z) \leq \int u^{*} d \mu \leq \int \varphi d \mu,
$$

which leads to a contradiction. Thus we get the equality. Clearly, $\overline{\mathcal{J}}_{z} \subset \widehat{\mathcal{J}}_{z}$. 
Suppose $\mu \in \widehat{\mathcal{J}}_{z}, z \in \bar{D}$. Then there exists a sequence $\mu_{j} \in \overline{\mathcal{J}}_{z_{j}}$ such that $z_{j} \in D, z_{j} \rightarrow z$ and $\mu_{j}$ converges weak-* to $\mu$. Take $u \in \mathrm{PSH}^{z}(D)$. Then for all $j, u\left(z_{j}\right) \leq \int u^{*} d \mu_{j}$. Using Lemma 2.2,

$$
\limsup _{j} u\left(z_{j}\right)=u(z) \leq \limsup _{j} \int u^{*} d \mu_{j} \leq \int u^{*} d \mu .
$$

Thus $\mu \in \mathcal{J}_{z}^{l}$ and we have proved $\widehat{\mathcal{J}}_{z} \subset \mathcal{J}_{z}^{l}$. The inclusion $\mathcal{J}_{z}^{l} \subset \mathcal{J}_{z}^{\mathrm{c}}$ is clear from the definition.

As the following example shows, in general, $\widehat{\mathcal{J}}_{z} \neq \overline{\mathcal{J}}_{z}$.

EXAmple 2.5. Consider this example due to Fornæss (see [K, Ex. 2.9.4]): Let $D \subset \mathbb{C}^{2}$ be the domain defined by

$$
D=[(U(0,2) \backslash \partial U) \times U] \cup \bigcup_{j=2}^{\infty} \partial U \times U\left(\frac{1}{j}, e^{e^{-j}}\right),
$$

where $U(a, r)$ is the disk in $\mathbb{C}$ centered at $a$ with radius $r$. We define a subharmonic function $\psi$ on $\mathbb{C}$ by

$$
\psi(w)=\sum_{j=2}^{\infty} \frac{2^{-j}}{\log j} \log \left|w-\frac{1}{j}\right|
$$

and a plurisubharmonic function $u$ on $D$ by

$$
u(z, w)= \begin{cases}\max \{\psi(w),-1\}, & |z|<1, \\ -1, & |z| \geq 1 .\end{cases}
$$

It was shown by Fornæss that there exists no sequence $u_{j} \in \mathrm{PSH}^{\mathrm{c}}(D)$ decreasing pointwise to $u$ on $D$. In particular, it is shown that $u$ does not satisfy the inequality

$$
u(0,0) \leq \sup _{|z|=3 / 2} u(z, 0) .
$$

Now we let $f_{j}(z)=(3 z / 2,1 / j)$ be the mappings of the closed unit disc $\bar{U}$ into $D$. Then $f_{j}(0)=z_{j}$ and $\mu_{f_{j}}$ is a Jensen measure in $\mathcal{J}_{z_{j}}(D)$ for each $j$. Note that $f_{j}$ converges to $f(z)=(3 z / 2,0)$ uniformly on $S=\{|z|=1\}$, so $\mu_{f_{j}}$ converges weak-* to $\mu_{f}$. Also note that $f$ maps $S$ into $D$ (but $f$ does not map $U$ into $D$ since $f(2 / 3)=(1,0) \notin D)$. Hence, if $z_{0}=(0,0)$, then $\mu_{f} \in \widehat{\mathcal{J}}_{z_{0}}$, but $\mu_{f} \notin \overline{\mathcal{J}}_{z_{0}}$ since $u$ does not satisfy $u\left(z_{0}\right) \leq \int u d \mu$.

3. Jensen multifunctions. Let $F$ and $G$ be topological spaces and let $p: F \times G \rightarrow F$ be the projection. A set $K \subset F \times G$ is a multifunction on $F$ if $p(K)=F$ and for each $x \in F$ the fiber $K_{x}=\{y \in G:(x, y) \in K\}$ is compact.

A multifunction $K$ is upper semicontinuous at $x \in F$ if for every neighborhood $V$ of $K_{x}$ in $F \times G$ there is a neighborhood $W$ of $x$ in $F$ such that 
$K_{y} \subset V$ when $y \in W$. A multifunction $K$ is lower semicontinuous at $x \in F$ if for every $(x, y) \in K_{x}$ and for every neighborhood $V$ of $(x, y)$ in $F \times G$ there is a neighborhood $W$ of $x$ in $F$ such that $K_{y} \cap V \neq \emptyset$ when $y \in W$.

We define the Jensen multifunction $\overline{\mathcal{J}}(D)$ as a set in $D \times C^{*}(\bar{D})$ with fibers $\overline{\mathcal{J}}_{z}(D)$ at $z \in D$. The multifunctions $\widehat{\mathcal{J}}(\bar{D}), \mathcal{J}^{\mathrm{b}}(\bar{D})$ and $\mathcal{J}^{\mathrm{c}}(\bar{D})$ are defined as sets in $\bar{D} \times C^{*}(\bar{D})$ with fibers $\widehat{\mathcal{J}}_{z}(\bar{D}), \mathcal{J}_{z}^{\mathrm{b}}(\bar{D})$ and $\mathcal{J}_{z}^{\mathrm{c}}(\bar{D})$ respectively at $z \in \bar{D}$.

If $m \geq 1$ is an integer and $r>0$, let $B_{r}$ be the closed ball in $\mathbb{R}^{m}$ centered at the origin and with radius $r$. We endow $\mathbb{R}^{m}$ with a scalar product $(x, y)$ and the norm $\|x\|$.

LEMma 3.1. Let $\left\{K_{n}\right\}$ be a sequence of non-empty convex and compact sets in $\mathbb{R}^{m}$ which are contained in $B_{R} \backslash B_{r}$ for some $R>r>0$. Then there exist a vector $u \in \mathbb{R}^{m}$ and a subsequence $\left\{K_{n_{k}}\right\}$ of $\left\{K_{n}\right\}$ so that for each $x \in K_{n_{k}}$ and each $k$ we have $(u, x) \geq 1$.

Proof. The sets $K_{n}$ and $B_{r}$ are disjoint convex sets. Hence for each $n$ there exists a number $\delta_{n}>0$ and a vector $v_{n} \in \mathbb{R}^{m}$ so that we have

$$
\left|\left(v_{n}, y\right)\right| \leq\left\|v_{n}\right\| r<\delta_{n}<\left(v_{n}, x\right)
$$

for all $x \in K_{n}$ and for all $y \in B_{r}$. Letting

$$
u_{n}=\frac{2 v_{n}}{\left\|v_{n}\right\| r}
$$

we have

$$
\left|\left(u_{n}, y\right)\right| \leq 2<\left(u_{n}, x\right)
$$

for all $x \in K_{n}$ and for all $y \in B_{r}$. Since $\left\|u_{n}\right\|=2 / r$, there exists a subsequence $\left\{u_{n_{k}}\right\}$ of $\left\{u_{n}\right\}$ that converges to a point $u \in \mathbb{R}^{m}$.

We take an integer $k_{0} \geq 1$ large enough so that $\left\|u-u_{n_{k}}\right\|<1 / R$ for all $k \geq k_{0}$. Then for any $x \in B_{R}$ and $k \geq k_{0}$,

$$
\left|\left(u-u_{n_{k}}, x\right)\right|<1 .
$$

In particular, for any $k \geq k_{0}$ and $x \in K_{n_{k}}$ we have

$$
(u, x)=\left(u_{n_{k}}, x\right)+\left(u-u_{n_{k}}, x\right) \geq 1 .
$$

Let $\varphi: \bar{D} \rightarrow \mathbb{R}$ be a continuous function. We let $B^{*}$ denote the closed unit ball in $C^{*}(\bar{D})$. If $K \subset \bar{D} \times C^{*}(\bar{D})$ is a multifunction on $D$ or $\bar{D}$ with fibers $K_{z} \subset B^{*}$, we set

$$
I \varphi(z)=\inf _{\mu \in K_{z}} \int \varphi d \mu, \quad S \varphi(z)=\sup _{\mu \in K_{z}} \int \varphi d \mu .
$$

THEOREM 3.2. The function $I \varphi$ (respectively $S \varphi$ ) is upper semicontinuous at a point $z_{0} \in \bar{D}$ for all $\varphi \in C(\bar{D})$ if the set $K$ is lower semicontinuous (respectively upper semicontinuous) at $z_{0}$. If the fibers $K_{z}$ are convex for 
$z \in \bar{D}$ (respectively if the fiber $K_{z_{0}}$ is convex), then the converse is also true.

Proof. (1) Suppose $K$ is lower semicontinuous at $z_{0}$. Choose $\mu \in K_{z_{0}}$ such that

$$
\int \varphi d \mu<I \varphi\left(z_{0}\right)+\frac{\varepsilon}{2}
$$

and let

$$
V=\left\{\mu+\nu:\left|\int \varphi d \nu\right|<\frac{\varepsilon}{2}, \nu \in C^{*}(\bar{D})\right\} .
$$

There exists a neighborhood $W$ of $z_{0}$ such that if $z \in W$ there exists $\mu+\nu_{z} \in$ $V \cap K_{z}$. Then

$$
I \varphi(z) \leq \int \varphi d \mu+\int \varphi d \nu_{z}<\int \varphi d \mu+\frac{\varepsilon}{2}<I \varphi\left(z_{0}\right)+\varepsilon .
$$

Hence $I \varphi$ is upper semicontinuous at $z_{0}$.

Now suppose $K$ is not lower semicontinuous at $z_{0} \in \bar{D}$. Then we can find an element $\mu \in K_{z_{0}}$, a neighborhood $V$ of $\mu$ and a sequence $z_{k} \in D$ such that $z_{k} \rightarrow z_{0}$ and $K_{z_{k}} \cap V=\emptyset$. We may assume that there are functions $\varphi_{j} \in C(\bar{D}), j=1, \ldots, m$, and a number $\varepsilon>0$ so that $V$ is of the form

$$
V=\left\{\nu \in C^{*}(\bar{D}):\left|\int \varphi_{j} d \mu-\int \varphi_{j} d \nu\right|<\varepsilon, j=1, \ldots, m\right\} .
$$

We set

$$
\psi_{j}=\varphi_{j}-\int \varphi_{j} d \mu
$$

Let

$$
C_{k}=\left\{\left(\int \psi_{1} d \nu, \ldots, \int \psi_{m} d \nu\right): \nu \in K_{z_{k}}\right\} .
$$

Since $K_{z_{k}}$ is compact and convex, the set $C_{k}$ is a compact convex subset of $\mathbb{R}^{m}$. Also note that since $K_{z_{k}} \cap V=\emptyset$, for every $\nu \in K_{z_{k}}$ there is $j \in$ $\{1, \ldots, m\}$ such that

$$
\left|\int \psi_{j} d \nu\right| \geq \varepsilon
$$

So $\|x\| \geq \varepsilon$ for every $x \in C_{k}$. Clearly, all sets $C_{k}$ are contained in $B_{R}$ for some $R>\varepsilon>0$. Now we can apply Lemma 3.1. There exist a vector $u=\left(u_{j}\right) \in \mathbb{R}^{m}$ and a subsequence of $\left\{K_{z_{k}}\right\}$, which we call $\left\{K_{z_{k}}\right\}$ again, so that

$$
\sum_{j=1}^{m} u_{j} \int \psi_{j} d \nu \geq 1
$$

for all $\nu \in K_{z_{k}}$ and for all $k$. If we set $\varphi=\sum_{j=1}^{m} u_{j} \psi_{j}$, then by (3.1) we get

$$
\int \varphi d \nu>1+\int \varphi d \mu
$$


for all $\nu \in K_{z_{k}}$ and for all $k$. Thus

$$
I \varphi\left(z_{0}\right) \leq \int \varphi d \mu<I \varphi\left(z_{k}\right)-1
$$

for a sequence $z_{k} \in D$ converging to $z_{0}$. Hence $I \varphi$ is not upper semicontinuous at $z_{0}$.

(2) Suppose $K$ is upper semicontinuous at $z_{0}$. Let

$$
V=K_{z_{0}}+\left\{\nu:\left|\int \varphi d \nu\right|<\varepsilon\right\}
$$

There exists a neighborhood $W$ of $z_{0}$ such that if $z \in W$ then $K_{z} \subset V$. Hence for all $\omega \in K_{z}$ there exists $\mu \in K_{z_{0}}$ such that

$$
\int \varphi d \mu+\varepsilon>\int \varphi d \omega>\int \varphi d \mu-\varepsilon
$$

Taking supremum over $\omega \in K_{z}$, we get

$$
S \varphi\left(z_{0}\right)+\varepsilon \geq S \varphi(z)
$$

for all $z \in W$. Thus $S \varphi$ is upper semicontinuous at $z_{0}$.

Suppose $K$ is not upper semicontinuous at some point $z_{0}$. There exist a sequence $\left\{w_{j}\right\} \subset D$ converging to $z_{0}$, a neighborhood $V$ of 0 in $C^{*}(\bar{D})$ and measures $\mu_{j} \in K_{w_{j}} \backslash\left(K_{z_{0}}+V\right)$. There exists a subsequence $\mu_{j_{k}}$ of $\mu_{j}$ that converges weak- $*$ to a measure $\mu \in B^{*}$. Then $\mu \notin K_{z_{0}}$ and once again by Theorem 2.3 there exist a function $\varphi \in C(\bar{D})$, an integer $k_{0} \geq 1$ and a number $a>0$ so that for all $k \geq k_{0}$,

$$
S \varphi\left(z_{0}\right)<\int \varphi d \mu-a<\int \varphi d \mu_{j_{k}} \leq S \varphi\left(z_{j_{k}}\right) .
$$

Thus $S \varphi$ is not upper semicontinuous at $z_{0}$.

Using Theorem 2.1 we obtain an immediate corollary of the above theorem:

Corollary 3.3. The set $\overline{\mathcal{J}}(D)$ is a lower semicontinuous multifunction in $D \times C^{*}(\bar{D})$.

We prove now that $\mathcal{J}^{\text {b }}$ is a lower semicontinuous multifunction. The second part of the following proposition was stated as a corollary of the Edwards' theorem in [W, Corollary 2.2], but it can also be seen directly from the first part.

Proposition 3.4. Let $D$ be a bounded domain in $\mathbb{C}^{n}$ and let $\varphi$ be a continuous function on $\bar{D}$. Then for every $z \in \bar{D}, E^{\mathrm{b}} \varphi(z)=(E \varphi)^{*}(z)$, i.e., $E^{\mathrm{b}} \varphi$ is upper semicontinuous on $\bar{D}$. Hence

$$
E^{\mathrm{b}} \varphi(z)=\sup \left\{u^{*}(z): u \in \operatorname{PSH}(D), u^{*} \leq \varphi\right\} .
$$

Proof. First let us prove that $E^{\mathrm{b}} \varphi$ is upper semicontinuous on $\bar{D}$. From Theorem 2.1 and Lemma 2.4, $E^{\mathrm{b}} \varphi=E \varphi$ is plurisubharmonic on $D$. Let 
$z \in \bar{D}$ and $\mu \in \mathcal{J}_{z}^{\mathrm{b}}$. Then

$$
\left(E^{\mathrm{b}} \varphi\right)^{*}(z) \leq \int\left(E^{\mathrm{b}} \varphi\right)^{*} d \mu \leq \int \varphi d \mu .
$$

Taking infimum over the measures $\mu \in \mathcal{J}_{z}^{\mathrm{b}}$ yields

$$
\left(E^{\mathrm{b}} \varphi\right)^{*}(z) \leq E^{\mathrm{b}} \varphi(z)
$$

hence $E^{\mathrm{b}} \varphi$ is upper semicontinuous. Since $E^{\mathrm{b}} \varphi=E \varphi$ on $D$,

$$
E^{\mathrm{b}} \varphi(z)=\left(E^{\mathrm{b}} \varphi\right)^{*}(z)=(E \varphi)^{*}(z)
$$

for all $z \in \bar{D}$. This yields the last assertion.

Corollary 3.5. The set $\mathcal{J}^{\mathrm{b}}(\bar{D})$ is a lower semicontinuous multifunction in $\bar{D} \times C^{*}(\bar{D})$.

This result allows us to to $\bar{D}$ extend an inclusion proved in Lemma 2.4.

Corollary 3.6. Let $z$ be a point in $\bar{D}, \mu \in \mathcal{J}_{z}^{\mathrm{b}}$ and $\left\{z_{j}\right\}$ be a sequence in $D$ converging to $z$. Then there are measures $\mu_{j} \in \overline{\mathcal{J}}_{z_{j}}$ that weak-* converge to $\mu$. Hence $\mathcal{J}_{z}^{\mathrm{b}} \subset \widehat{\mathcal{J}}_{z}$ for all $z \in \bar{D}$.

Proof. Since $C(\bar{D})$ is separable, the weak-* topology on $\mathcal{M}(\bar{D})$ is metrizable by some metric (see [C, Theorem 5.1]). If $\mu \in \mathcal{J}_{z}^{\mathrm{b}}$, let $\beta_{k}$ be the open ball of radius $1 / k$ around $\mu$ in this metric. Since $\beta_{k}$ is open and $\mathcal{J}^{\mathrm{b}}$ is a lower semicontinuous multifunction, for any sequence $z_{j} \in D$ converging to $z$ there exist $j_{k} \geq 1$ and $\mu_{j} \in \beta_{k} \cap \overline{\mathcal{J}}_{z_{j}}$ for all $j \geq j_{k}$. Then the sequence $\left\{\mu_{j}\right\}$ converges to $\mu$ and $\mu \in \widehat{\mathcal{J}}_{z}$.

THEOREM 3.7. The sets $\widehat{\mathcal{J}}(\bar{D})$ and $\mathcal{J}^{\mathrm{c}}(\bar{D})$ are upper semicontinuous multifunctions in $\bar{D} \times C^{*}(\bar{D})$.

Proof. Let $K$ be either $\widehat{\mathcal{J}}(\bar{D})$ or $\mathcal{J}^{\mathrm{c}}(\bar{D})$. Suppose $K$ is not upper semicontinuous at $z_{0} \in \bar{D}$. There exist a neighborhood $V$ of $K_{z_{0}}$, a sequence of points $z_{j} \in \bar{D}$ and a sequence of measures $\mu_{j} \in K_{z_{j}}$ such that $z_{j} \rightarrow z_{0}$ and $\mu_{j} \notin V$. Since $K$ is compact, we can choose a weak-* convergent subsequence $\mu_{j_{k}}$. Let $\mu=\lim \mu_{j_{k}}$. It is easy to see that $\mu \in K_{z_{0}}$, hence $\mu \in V$. This leads to a contradiction since $\mu_{j_{k}} \notin V$.

As we prove in the next theorem, the envelope $\widehat{E} \varphi$ coincides with the lower semicontinuous regularization of $E \varphi$ on $\bar{D}$. If $\psi$ is a bounded function on $D$ we define its lower semicontinuous regularization $\psi_{*}$ on $\bar{D}$ as

$$
\psi_{*}(z)=\liminf _{w \rightarrow z, w \in D} \psi(w) .
$$

TheOREm 3.8. If $\varphi \in C(\bar{D})$, then for all $z \in \bar{D}$,

$$
\widehat{E} \varphi(z)=(E \varphi)_{*}(z)=\inf \left\{\int \varphi d \mu: \mu \in \widehat{\mathcal{J}}_{z}\right\} .
$$


Proof. Take $z \in \bar{D}$ and suppose

$$
E \varphi\left(z_{j}\right)<\widehat{E} \varphi(z)-\varepsilon
$$

for some sequence of points $z_{j} \in D$ such that $z_{j} \rightarrow z$ and some number $\varepsilon>0$. We can find a sequence $\mu_{j} \in \overline{\mathcal{J}}_{z_{j}}$ so that for all $j$,

$$
\int \varphi d \mu_{j}<\widehat{E} \varphi(z)-\varepsilon .
$$

There exists a subsequence $\mu_{j_{k}}$ such that $\mu_{j_{k}} \rightarrow \mu$ for some $\mu \in \widehat{\mathcal{J}}_{z}$. Hence, letting $j_{k} \rightarrow \infty$ yields

$$
\int \varphi d \mu \leq \widehat{E} \varphi(z)-\varepsilon
$$

On the other hand, $\widehat{E} \varphi(z) \leq \int \varphi d \nu$ for all $\nu \in \widehat{\mathcal{J}}_{z}$, which gives

$$
\int \varphi d \mu \leq \widehat{E} \varphi(z)-\varepsilon \leq \int \varphi d \mu-\varepsilon,
$$

a contradiction. Thus $(E \varphi)_{*}(z) \geq \widehat{E} \varphi(z)$.

Suppose $\widehat{E} \varphi(z)+\varepsilon<(E \varphi)_{*}(z)$ for some point $z \in \bar{D}$ and some number $\varepsilon>0$. We may find an element $\mu$ of $\widehat{\mathcal{J}}_{z}$ so that

$$
\int \varphi d \mu \leq \widehat{E} \varphi(z)+\varepsilon
$$

There exist a sequence $z_{j} \in D$ and $\mu_{j} \in \overline{\mathcal{J}}_{z_{j}}$ such that $z_{j} \rightarrow z$ and $\mu_{j}$ converges weak- $*$ to $\mu$. Since $E \varphi\left(z_{j}\right) \leq \int \varphi d \mu_{j}$ for all $j$,

$$
\begin{aligned}
(E \varphi)_{*}(z) & \leq \liminf _{j} E \varphi\left(z_{j}\right) \leq \lim _{j} \int \varphi d \mu_{j}=\int \varphi d \mu \\
& \leq \widehat{E} \varphi(z)+\varepsilon<(E \varphi)_{*}(z) .
\end{aligned}
$$

This contradiction proves the equality.

\section{4. $C$-regular boundaries and continuity of plurisubharmonic en-} velopes. Our goal in this section is to relate Jensen measures to the continuity of plurisubharmonic envelopes. We begin with some terminology.

Definition 4.1. Let $\Omega$ be a bounded open subset of $\mathbb{C}^{n}$. A point $z \in \bar{\Omega}$ is said to be $c$-regular if the following holds: If $z_{j} \in D, z_{j} \rightarrow z$ and $\mu \in \widehat{\mathcal{J}}_{z}$, then there exists a sequence $\mu_{j} \in \overline{\mathcal{J}}_{z_{j}}$ that converges weak-* to $\mu$. The boundary $\partial \Omega$ of $\Omega$ is said to be $c$-regular if all boundary points are $c$-regular.

We say that the plurisubharmonic envelope $E \varphi$ of a function $\varphi$ defined on $\bar{D}$ is continuous at a point $z \in \bar{D}$ if $(E \varphi)^{*}(z)=(E \varphi)_{*}(z)$. The main theorem of this section proves that $c$-regular points are exactly those where the plurisubharmonic envelopes are continuous.

TheOREM 4.2. Let $D$ be a bounded domain in $\mathbb{C}^{n}$ and $z$ be a point in $\bar{D}$. Then the following statements are equivalent: 
(1) The envelope $E \varphi$ is continuous at $z$ for all functions $\varphi \in C(\bar{D})$.

(2) $\mathcal{J}_{z}^{\mathrm{b}}=\mathcal{J}_{z}^{l}$.

(3) $\mathcal{J}_{z}^{\mathrm{b}}=\widehat{\mathcal{J}}_{z}$.

(4) The point $z$ is c-regular.

Proof. First we note that in view of Lemma 2.4, (2) implies (3). Then suppose the envelopes $E \varphi$ are continuous at a point $z \in \bar{D}$ for every function $\varphi \in C(\bar{D})$. We already know from Lemma 2.4 and Corollary 3.6 that

$$
\mathcal{J}_{z}^{\mathrm{b}} \subset \widehat{\mathcal{J}}_{z} \subset \mathcal{J}_{z}^{l} \text {. }
$$

Suppose there exists a measure $\mu \in \mathcal{J}_{z}^{l} \backslash \mathcal{J}_{z}^{\mathrm{b}}$. Then using Theorem 2.3 one can find a function $\varphi \in C(\bar{D})$ and a number $a>0$ so that

$$
\int \varphi d \mu<E^{\mathrm{b}} \varphi(z)-a .
$$

But now by Proposition $3.4, E^{\mathrm{b}} \varphi$ is in $\mathrm{PSH}^{z}(D)$, therefore

$$
E^{\mathrm{b}} \varphi(z) \leq \int \varphi d \mu<E^{\mathrm{b}} \varphi(z)-a .
$$

Thus we have equality in (4.1). Then Corollary 3.6 implies that the point $z$ is $c$-regular. Hence we have shown (1) implies (2) and (4).

If $\mathcal{J}_{z}^{\mathrm{b}}=\widehat{\mathcal{J}}_{z}$, then $z$ is $c$-regular in view of Corollary 3.6. Using Proposition 3.4 and Theorem 3.8 we see that $(E \varphi)^{*}(z)=(E \varphi)_{*}(z)$, hence $E \varphi$ is continuous at $z$. Thus (3) implies (4) and (1).

Next we show that (4) implies (1) to finish the proof. Suppose $z$ is $c$ regular and suppose there exist a sequence $\left\{z_{j}\right\} \subset D$ and a number $a>0$ such that $z_{j} \rightarrow z$ and

$$
E \varphi\left(z_{j}\right)>\widehat{E} \varphi(z)+a .
$$

Hence there exists an element $\mu \in \widehat{\mathcal{J}}_{z}$ such that for any sequence $\mu_{j} \in \overline{\mathcal{J}}_{z_{j}}$,

$$
\int \varphi d \mu_{j} \geq E \varphi\left(z_{j}\right)>\widehat{E} \varphi(z)+a>\int \varphi d \mu .
$$

Since $z$ is $c$-regular, there is a sequence $\left\{\nu_{j}\right\} \subset \overline{\mathcal{J}}_{z_{j}}$ that converges weak-* to $\mu \in \widehat{\mathcal{J}}_{z}$. Therefore letting $j \rightarrow \infty$ leads to

$$
\int \varphi d \mu \geq \widehat{E} \varphi(z)+a>\int \varphi d \mu
$$

a contradiction. Hence $(E \varphi)^{*}(z) \leq \widehat{E} \varphi(z)$. From Theorem 3.8, $\widehat{E} \varphi(z)=$ $(E \varphi)_{*}(z)$, so $(E \varphi)_{*}(z)=(E \varphi)^{*}(z)$. Thus (4) implies (1).

Using the following observation from [Wa, Lemma 1], it is enough to prove the continuity of the plurisubharmonic envelope on the boundary if we want to prove that the envelope is continuous on $\bar{D}$. 
Theorem 4.3. Let $D$ be a bounded domain in $\mathbb{C}^{n}$ and let $g: \bar{D} \rightarrow$ $[-\infty, \infty]$ have the property that its restriction to $D$ is either uniformly continuous or identically $+\infty$. If $(E g)^{*}$ is dominated by $g$ on $\bar{D}$ and continuous on $\partial D$, then $E g$ is continuous in $D$.

In the terminology of [W], a bounded domain $D$ in $\mathbb{C}^{n}$ is said to have the approximation property if for each $u \in \operatorname{PSH}^{\mathrm{b}}(D)$ there exist $u_{j} \in \mathrm{PSH}^{\mathrm{c}}(D)$ such that $u_{j}(z) \downarrow u^{*}(z)$ for all $z \in \bar{D}$. It was shown in [W] that $\mathcal{J}_{z}^{\mathrm{c}}=\mathcal{J}_{z}^{\mathrm{b}}$ for all $z \in \bar{D}$ if and only if $D$ has the approximation property. We may now use Theorems 4.2 and 4.3 to prove the following.

Corollary 4.4. Let $D$ be a bounded domain in $\mathbb{C}^{n}$. Then the following statements are equivalent:

(1) E $\varphi$ is continuous on $\bar{D}$ for all functions $\varphi \in C(\bar{D})$.

(2) $\mathcal{J}_{z}^{\mathrm{b}}=\mathcal{J}_{z}^{\mathrm{c}}$ for all $z \in \partial D$.

(3) $\mathcal{J}_{z}^{\mathrm{b}}=\widehat{\mathcal{J}}_{z}$ for all $z \in \partial D$.

(4) $\partial D$ is c-regular.

(5) D has the approximation property.

Proof. The equivalence of (1), (3) and (4) follows from Theorems 4.2 and 4.3. By $[\mathrm{W}],(5)$ implies (2). We already know by Lemma 2.4 that $\mathcal{J}_{z}^{\mathrm{b}} \subset \widehat{\mathcal{J}}_{z} \subset \mathcal{J}_{z}^{\mathrm{c}}$. Thus (2) implies (3). We show that (1) implies (2) and (5) to finish the proof.

Thus suppose the envelopes are continuous on $\bar{D}$. Let $z \in \bar{D}$. Suppose there exists a measure $\mu \in \mathcal{J}_{z}^{\mathrm{c}} \backslash \mathcal{J}_{z}^{\mathrm{b}}$. Then by Theorem 2.3 there exist a function $\varphi \in C(\bar{D})$ and a number $a>0$ such that

$$
\int \varphi d \mu \leq \inf \left\{\int \varphi d \nu: \nu \in \mathcal{J}_{z}^{\mathrm{b}}\right\}-a=E^{\mathrm{b}} \varphi(z)-a .
$$

Since $\mu \in \mathcal{J}_{z}^{\mathrm{c}}$ and since $E^{\mathrm{b}} \varphi$ is continuous on $\bar{D}$,

$$
E^{\mathrm{b}} \varphi(z) \leq \int \varphi d \mu \leq E^{\mathrm{b}} \varphi(z)-a,
$$

which is a contradiction. Thus $\mathcal{J}_{z}^{\mathrm{c}}=\widehat{\mathcal{J}}_{z}=\mathcal{J}_{z}^{\mathrm{b}}$ for all $z \in \bar{D}$. This implies (2), and (5) follows from [W].

5. Continuity of the Perron-Bremermann function. This section is devoted to the Perron-Bremermann function. For any function $f$ on $\partial D$ we let

$$
\mathcal{S} f(z)=\sup \left\{v^{*}(z): v \in \operatorname{PSH}(D),\left.v^{*}\right|_{\partial D} \leq f\right\}
$$

for all $z \in \bar{D}$. If $f \in C(\partial D)$, then the upper semicontinuous regularization $\mathcal{S}^{*} f$ of the map $\mathcal{S} f$ defined on $\bar{D}$ is called the Perron-Bremermann function of $f$ on $\bar{D}$ (see $[\mathrm{K}]$ ). The following lemma is useful: 
LEMMA 5.1. Suppose $s$ is an upper semicontinuous function on $\bar{D}$ and $f \in C(\partial D)$ satisfies $s(\zeta) \leq f(\zeta)$ for all $\zeta \in \partial D$. Then there exists a continuous extension $\varphi$ of $f$ on $\bar{D}$ such that $s \leq \varphi$ on $\bar{D}$.

Proof. We may find a sequence of exhausting subdomains $V_{j}$ so that $\bigcup_{j} V_{j}=D$ and $\bar{V}_{j} \subset V_{j+1}$. For each $j$ we take a function $\chi_{j} \in C(\bar{D})$ such that $0 \leq \chi_{j} \leq 1$ and

$$
\chi_{j}= \begin{cases}1 & \text { on } \bar{V}_{j}, \\ 0 & \text { on } \bar{D} \backslash V_{j+1} .\end{cases}
$$

We extend $f$ as a continuous function $F$ on $\bar{D}$. Now the function $r=$ $\max \{s, F\}$ is upper semicontinuous. There exist functions $r_{j} \in C(\bar{D})$ such that $r_{j}(z)$ decreases to $r(z)$ for every $z \in \bar{D}$. We define a function $\psi$ on $D$ as follows: Given any $z \in D$, there is a unique integer $j \geq 1$ such that $z \in \bar{V}_{j+1} \backslash \bar{V}_{j}$. Set

$$
\psi(z)=\chi_{j}(z) r_{j}(z)+\left(1-\chi_{j}(z)\right) r_{j+1}(z) .
$$

Now, evidently, the function $\psi$ is continuous on $D$. For all $z \in D$,

$$
r(z) \leq r_{j+1}(z) \leq \psi(z) \leq r_{j}(z) .
$$

Let $\varphi$ be equal to $\max \{\psi, F\}$ on $D$ and equal to $f$ on $\partial D$. Then $\varphi \geq s$ on $\bar{D}$. We only need to show that $\varphi$ is continuous on $\bar{D}$.

Clearly $\varphi$ is continuous on $D$. Let $z_{j} \in \bar{D}$ be such that $z_{j} \rightarrow \zeta \in \partial D$. Then

$$
\liminf _{j} \varphi\left(z_{j}\right) \geq \liminf _{j} F\left(z_{j}\right)=F(\zeta)=f(\zeta)=\varphi(\zeta) .
$$

Hence $\varphi$ is lower semicontinuous on $\bar{D}$. Since $\left.r\right|_{\partial D}=f$ is continuous, the functions $r_{j}$ decrease uniformly to $r$ on $\partial D$. Thus for a given number $\varepsilon>0$, one can find an integer $j_{0} \geq 1$ such that

$$
r_{j}(\zeta)<f(\zeta)+\varepsilon=F(\zeta)+\varepsilon
$$

for all points $\zeta \in \partial D$ and integers $j \geq j_{0}$. Since $r_{j_{0}}$ and $F$ are uniformly continuous on $\bar{D}$, we can find a number $\delta>0$ so that

$$
r_{j_{0}}(z)<F(z)+\varepsilon
$$

for all $z \in D_{\delta}$, where

$$
D_{\delta}=\{z \in \bar{D}: \operatorname{dist}(z, \partial D) \leq \delta\} .
$$

We can find an integer $k_{0} \geq 1$ so that $\bar{D} \backslash \bar{V}_{k} \subset D_{\delta}$ for all integers $k \geq k_{0}$. Let $k$ be an integer greater than or equal to $\max \left\{k_{0}, j_{0}\right\}$. By (5.1) and (5.2),

$$
\psi(z)<F(z)+\varepsilon
$$

for each $z \in D \backslash \bar{V}_{k}$. Thus for each $\varepsilon>0$ we can find an integer $k \geq 1 \mathrm{such}$ that $\varphi(z)<F(z)+\varepsilon$ for all $z \in \bar{D} \backslash \bar{V}_{k}$. This implies that $\varphi$ is also upper semicontinuous on $\bar{D}$. 
The above lemma and Proposition 3.4 allow us to describe the function $\mathcal{S} f$ as in the next result.

Proposition 5.2. Let $D$ be a bounded domain in $\mathbb{C}^{n}$ and let $f \in C(\partial D)$. Then the function $\mathcal{S} f$ coincides with the function

$$
\mathcal{U} f(z)=\sup \left\{E^{\mathrm{b}} \varphi(z): \varphi \in C(\bar{D}),\left.\varphi\right|_{\partial D}=f\right\}
$$

on $\bar{D}$.

Proof. In view of Theorem 2.1 and Proposition 3.4, $\mathcal{S} f \geq \mathcal{U} f$ on $\bar{D}$. To get the reverse inequality let $v \in \operatorname{PSH}(D)$ with $v^{*} \leq f$ on $\partial D$. By Lemma 5.1 there exists a function $\varphi \in C(\bar{D})$ such that $\varphi=f$ on $\partial D$ and $v^{*} \leq \varphi$ on $\bar{D}$. Therefore $\mathcal{S} f \leq \mathcal{U} f$ on $\bar{D}$, which implies the equality $\mathcal{S} f=\mathcal{U} f$.

As a consequence of Proposition 5.2 and Theorem 4.2 we get the following.

Corollary 5.3. Let $D$ be a bounded domain in $\mathbb{C}^{n}, z \in \bar{D}$ and let $f \in C(\partial D)$. Then $\mathcal{S} f$ is lower semicontinuous at the point $z$. In particular, if $D$ is c-regular, then the function $\mathcal{S} f$ is lower semicontinuous on $\bar{D}$.

Now let us prove an integral representation of the function $\mathcal{S} f$.

Proposition 5.4. Let $f \in C(\partial D)$. For every integer $k \geq 0$ let $f_{k}$ be defined as $k$ on $D$ and $f$ on $\partial D$. Then there exists an integer $k_{0}$ such that for all $z \in \bar{D}$ and for all $k \geq k_{0}$,

$$
\mathcal{S} f(z)=\inf \left\{\int f_{k} d \mu: \mu \in \mathcal{J}_{z}^{\mathrm{b}}\right\} .
$$

Moreover, for all $z \in \bar{D}$ there exists $\mu_{z} \in \mathcal{J}_{z}^{\mathrm{b}}$ with $\operatorname{supp} \mu_{z} \subset \partial D$ such that $\mathcal{S} f(z)=\int_{\partial D} f d \mu_{z}$.

Proof. We shall suppose $f \leq 0$ on $\partial D$ and extend $f$ as a lower semicontinuous function $f_{k}$ on $\bar{D}$ by defining $f_{k}=k$ on $D$ where $k \geq 0$ is an integer. We let

$$
s(z)=\sup \left\{v^{*}(z): v \in \operatorname{PSH}^{\mathrm{b}}(D), v^{*} \leq f_{k} \text { on } \bar{D}\right\} .
$$

Clearly $s(z) \leq \mathcal{S} f(z)$. If $v \in \operatorname{PSH}^{\mathrm{b}}(D)$ and $v^{*} \leq f$ on $\partial D$, then $v^{*} \leq f_{k}$ on $\bar{D}$. Thus we get $\mathcal{S}(z)=s(z)$. It follows from Edwards' theorem (see for instance [W, Theorem 2.1] for a general version) that

$$
s(z)=\inf \left\{\int f_{k} d \mu: \mu \in \mathcal{J}_{z}^{\mathrm{b}}\right\}
$$

for all $z \in \bar{D}$.

For the second part, we first prove that for all $k \geq 0$ there exists $\mu_{k} \in \mathcal{J}_{z}^{\mathrm{b}}$ such that

$$
\mathcal{S} f(z)=\int_{\bar{D}} f_{k} d \mu_{k}
$$


For each $k \geq 0$, there exist measures $\nu_{j} \in \mathcal{J}_{z}^{\mathrm{b}}$ such that

$$
\mathcal{S} f(z) \leq \int f_{k} d \nu_{j}<\mathcal{S} f(z)+\frac{1}{j} .
$$

Since $\mathcal{J}_{z}^{\mathrm{b}}$ is closed, there exist a subsequence of $\left\{\nu_{j}\right\}$, which we denote by $\left\{\nu_{j}\right\}$ again, and $\nu \in \mathcal{J}_{z}^{\mathrm{b}}$ such that $\nu_{j} \rightarrow \nu$ weak-*. By the first part proved above, $\mathcal{S} f(z) \leq \int f_{k} d \nu$. Also $\liminf \operatorname{in}_{j \rightarrow \infty} \int f_{k} d \nu_{j}=\mathcal{S} f(z) \geq \int f_{k} d \nu$. Thus we take $\mu_{k}=\nu$.

Now a subsequence of $\left\{\mu_{k}\right\}$, say $\left\{\mu_{k}\right\}$ again, converges weak-* to some $\mu \in \mathcal{J}_{z}^{\mathrm{b}}$. Suppose there exist a compact set $K \subset D$ and a number $c>0$ such that for all $k$,

$$
\mu_{k}(K)>c .
$$

Let $m=\min _{\partial D} f$. Then

$$
\mathcal{S} f(z)=\int_{\bar{D}} f_{k} d \mu_{k} \geq m+k c
$$

Hence $\mathcal{S} f(z)=\infty$, which is impossible. Therefore for any compact set $K \subset D$, $\mu_{k}(K) \rightarrow 0$ as $k \rightarrow \infty$. This implies $\operatorname{supp} \mu \subset \partial D$.

For each $k$,

$$
\mathcal{S} f(z)=\int_{\bar{D}} f_{k} d \mu_{k} \geq \int_{\bar{D}} f_{0} d \mu_{k} .
$$

Also since $f_{0}$ is lower semicontinuous,

$$
\liminf _{k} \int_{\bar{D}} f_{0} d \mu_{k} \geq \int_{\bar{D}} f_{0} d \mu \geq \mathcal{S} f_{0}(z)=\mathcal{S} f(z),
$$

hence

$$
\mathcal{S} f(z)=\int_{\partial D} f_{0} d \mu
$$

This completes the proof.

If $A$ is any subset of $\bar{D}$, the characteristic function of $A$, which is equal to 1 if $z \in A$ and 0 if $z \notin A$, is denoted by $\chi_{A}(z)$.

Definition 5.5. A point $z_{0} \in \partial D$ is called a regular point if for every neighborhood $V$ of $z_{0}$ in $\bar{D}$,

$$
\limsup _{z \rightarrow z_{0}, z \in V \cap D} \mathcal{S}\left(-\left.\chi_{V \cap \partial D}\right|_{\partial D}\right)(z)=-1 .
$$

A domain $D$ is called a regular domain if every boundary point of $D$ is a regular point.

TheOREm 5.6. Let $D$ be a regular and c-regular domain. Then for any continuous function $f \in C(\partial D)$ the function $\mathcal{S} f$ is continuous on $\bar{D}$. 
Proof. Take a point $\zeta \in \partial D$ and a function $f \in C(\partial D)$. We may assume that $f<0$ on $\partial D$ and $f(\zeta)=-1$. For a given number $\varepsilon>0$ let

$$
W=\{w \in \partial D:|f(w)-f(\zeta)|<\varepsilon\} .
$$

Then $f \leq-\chi_{W}+\varepsilon$ on $\partial D$ and

$$
\mathcal{S}^{*} f(\zeta) \leq \limsup _{z \rightarrow \zeta, z \in D} \mathcal{S}\left(-\chi_{W}+\varepsilon\right)(z)=f(\zeta)+\varepsilon
$$

Since this holds for all $\varepsilon>0$ we get the inequality

$$
\mathcal{S}^{*} f(\zeta) \leq f(\zeta)
$$

for all $\zeta \in \partial D$. Thus $\mathcal{S}^{*} f(\zeta)=\mathcal{S} f(\zeta)$ and $\mathcal{S} f$ is upper semicontinuous on $\bar{D}$. By Corollary 5.3 the function $\mathcal{S} f$ is lower semicontinuous on $\bar{D}$. This proves the continuity of $\mathcal{S} f$.

Let us illustrate with the following example why we may need the assumption that the boundary should be regular.

Example 5.7. Let $D=U \backslash\{0\}$ be the punctured unit disk in $\mathbb{C}$. Since the plurisubharmonic envelopes on $\bar{D}$ are the same as those on $\bar{U}$, the domain $D$ is $c$-regular. Let $f(0)=-1$ and $f(z)=0$ for any $z \in \partial U$. Then $f \in C(\partial D)$ while $\mathcal{S} f$ is not continuous on $\bar{D}$.

We will need the following result from [CCW, Theorem 2.8].

Theorem 5.8. A bounded domain $D$ in $\mathbb{C}^{n}$ is hyperconvex if and only if for every $z \in \partial D$ and every measure $\mu \in \mathcal{J}_{z}^{\mathrm{c}}$, supp $\mu \subset \partial D$.

The above characterization of hyperconvex domains implies the following.

Theorem 5.9. Let $D$ be a hyperconvex domain in $\mathbb{C}^{n}$. If the envelope $\mathcal{S} f$ is continuous on $\bar{D}$ for every $f \in C(\partial D)$, then $D$ is c-regular.

Proof. Take a function $\varphi \in C(\bar{D})$ and let $f$ be the restriction of $\varphi$ to $\partial D$. We may assume that $\varphi \leq 0$ on $\partial D$. For any measure $\mu \in \mathcal{J}_{z}^{\mathrm{b}}$, where $z \in \partial D$, we have

$$
\int_{\bar{D}} \varphi d \mu=\int_{\partial D} f d \mu
$$

by Theorem 5.8. Thus by Propositions 5.2 and $5.4, E^{\mathrm{b}} \varphi(z)=\mathcal{S} f(z)$ for all $z \in \partial D$. This means that $E^{\mathrm{b}} \varphi$ is continuous on $\partial D$. Note that by Proposition 3.4, $E^{\mathrm{b}} \varphi=(E \varphi)^{*}$. According to Theorem 4.3 the envelope $E^{\mathrm{b}} \varphi$ is continuous on $\bar{D}$. This completes the proof.

6. Strongly regular points. In this section we characterize strongly regular points in terms of Jensen measures. The following definition is from [P2]: 
Definition 6.1. A point $z \in \partial D$ is called strongly regular if for every number $t>0$ there exists a neighborhood $V$ of $z$ and a negative plurisubharmonic function $v$ on $V_{1}=V \cap D$ such that the closure of the ball $B(z, 1 / t)$ is contained in $V, v<-\varepsilon<0$ on $V_{1} \backslash B(z, 1 / t)$ and $v(w) \rightarrow 0$ as $w \rightarrow z$ in $D$.

REMark 6.2. Since the function equal to $\max \{v,-\varepsilon\}$ on $V_{1}$ and $-\varepsilon$ on $D \backslash V_{1}$ is plurisubharmonic on $D$, we may assume in this definition that $v$ is defined globally on $D$.

TheOREM 6.3. Let $D$ be a bounded domain in $\mathbb{C}^{n}$ and $z \in \partial D$. The point $z$ is strongly regular if and only if $\widehat{\mathcal{J}}_{z}=\left\{\delta_{z}\right\}$.

Proof. Suppose the point $z \in \partial D$ is strongly regular. By a result of Poletsky ([P1, Lemma 8.2]), the plurisubharmonic envelope $E \varphi$ of any function $\varphi \in C(\bar{D})$ satisfies

$$
\lim _{w \rightarrow z, w \in D} E \varphi(w)=\varphi(z) .
$$

Hence the envelopes are continuous at $z$. Then $\widehat{\mathcal{J}}_{z}=\mathcal{J}_{z}^{\mathrm{b}}$ by Theorem 4.2. Let $\mu \in \mathcal{J}_{z}^{\mathrm{b}}$. Suppose for some numbers $t>0$ and $c>0, \mu\left(V_{t}\right)>c$, where $V_{t}=\bar{D} \backslash B(z, 1 / t)$. Since $z$ is strongly regular, there exist a plurisubharmonic function $v$ on $D$ and a number $\varepsilon>0$ such that $\lim _{w \rightarrow z, w \in D} v(w)=0$ and $v^{*}(w) \leq-\varepsilon$ for all $w \in V_{t}$. Then

$$
0=v^{*}(z) \leq \int_{\bar{D}} v^{*} d \mu=\int_{V_{t}} v^{*} d \mu+\int_{\bar{D} \backslash V_{t}} v^{*} d \mu<-c \varepsilon<0 .
$$

This implies that $\mu\left(V_{t}\right)=0$ for all $t>0$, therefore $\mu=\delta_{z}$ and $\widehat{\mathcal{J}}_{z}=\mathcal{J}_{z}^{\mathrm{b}}$ $=\left\{\delta_{z}\right\}$.

Conversely, suppose $\widehat{\mathcal{J}}_{z}=\left\{\delta_{z}\right\}$. Then also $\mathcal{J}_{z}^{\mathrm{b}}=\left\{\delta_{z}\right\}$. Choose a function $\varphi \in C(\bar{D})$ such that $\varphi(z)=0$ and $\varphi(w)<0$ for any $w \in \bar{D} \backslash\{z\}$. Then $(E \varphi)^{*}(z)=E^{\mathrm{b}} \varphi(z)=0$. To show that the limit exists at $z$, suppose that there exist a sequence of points $\left\{z_{j}\right\} \subset D$ converging to $z$ and a number $a>0$ such that $E \varphi\left(z_{j}\right)<-a<0$. We can find measures $\mu_{j} \in \overline{\mathcal{J}}_{z_{j}}$ such that

$$
\int \varphi d \mu_{j}<-a \text {. }
$$

A subsequence $\left\{\mu_{j_{k}}\right\}$ converges weak-* to a measure $\mu$. Then $\mu \in \widehat{\mathcal{J}}_{z}$, therefore $\mu=\delta_{z}$. Finally, we get

$$
0=\varphi(z)<-a,
$$

a contradiction. Thus the limit of $E \varphi$ exists at the point $z$.

If $t>0$, then there exists a number $\varepsilon>0$ so that $\varphi(w)<-\varepsilon$ for any $w \in \bar{D} \backslash B(z, 1 / t)$. Since $E^{\mathrm{b}} \varphi \leq \varphi$ on $\bar{D}, E^{\mathrm{b}} \varphi$ satisfies the requirements in Definition 6.1. 
In the terminology of [S] a domain $D$ is $B$-regular if the Dirichlet problem for continuous data on the boundary has a solution, that is, for any function $f \in C(\partial D)$ there exists a function $u \in \operatorname{PSH}^{\mathrm{c}}(D)$ so that $\left.u\right|_{\partial D}=f$. E. Poletsky (see [P1, Lemma 8.1]) proved that a boundary point is strongly regular if and only if at this point there exists a negative plurisubharmonic barrier. This means, for example, that a domain is strongly regular if and only if it is $B$-regular. It is shown in $[\mathrm{S}]$ that a domain $D$ is $B$-regular if and only if $J_{z}^{\mathrm{c}}=\left\{\delta_{z}\right\}$ for any $z \in \partial D$ (see also [W, Corollary 3.8]). We can now combine these results with Theorem 6.3 in one corollary:

Corollary 6.4. Let $D$ be a domain in $\mathbb{C}^{n}$. Then the following are equivalent:

(1) $D$ is strongly regular.

(2) $D$ is B-regular.

(3) For any $z \in \partial D, \mathcal{J}_{z}^{\mathrm{c}}=\left\{\delta_{z}\right\}$.

(4) For any $z \in \partial D, \widehat{\mathcal{J}}_{z}=\left\{\delta_{z}\right\}$.

For a bounded domain $D$, denote the set of strongly regular points of $D$ by $\mathcal{R}$. As shown by Example 7.3 below, the set $\mathcal{R}$ need not be a closed subset of the boundary in general. As a corollary of Theorem 6.3 we obtain:

COROLlaRY 6.5. If $D$ is c-regular, then the set of strongly regular points is a closed subset of $\partial D$.

Proof. Take a sequence of strongly regular points $z_{j} \in \mathcal{R}$ that converges to a point $z \in \partial D$. From Theorem $6.3, \mathcal{J}_{z_{j}}^{\mathrm{b}}=\widehat{\mathcal{J}}_{z_{j}}=\left\{\delta_{z_{j}}\right\}$ for each $j$. If $D$ is $c$-regular, then by Theorem $4.2, \widehat{\mathcal{J}}_{z}=\mathcal{J}_{z}^{\mathrm{b}}$, and by Corollary 3.6, for each measure $\mu \in \widehat{\mathcal{J}}_{z}$ there exists a sequence of measures $\mu_{j} \in \mathcal{J}_{z_{j}}^{\mathrm{b}}$ that converges weak-* to $\mu$. Since $\mu_{j}=\delta_{z_{j}}$ for all $j$, we have $\mu=\delta_{z}$. Hence $\widehat{\mathcal{J}}_{z}=\left\{\delta_{z}\right\}$ and $z$ is strongly regular by Theorem 6.3 .

7. An example. In this section we present an example of a strongly hyperconvex, star-shaped domain $D$ with smooth boundary that is not $c$ regular. Also on the boundary $\partial D$ of this domain there exists a function $f \in C(\partial D)$ so that $\mathcal{S} f$ is not continuous on $\partial D$.

Definition 7.1. A domain $D \subset \mathbb{C}^{n}$ is said to be star-shaped with respect to $0 \in \mathbb{C}^{n}$ if for all $t \in[0,1], t D \subset D$.

In [W, Thm. 4.10], it is shown that on star-shaped domains, $\mathcal{J}_{z}^{\mathrm{c}}=\mathcal{J}_{z}^{\mathrm{b}}$ for all $z \in D$. It follows from Theorems 3.2, 3.7 and 4.2 that if $D$ is starshaped, then the plurisubharmonic envelopes of continuous functions on $\bar{D}$ are continuous on $D$. So it is natural to ask if the envelopes are continuous up to the boundary. The answer is in general no, as the example of this section shows. 
We will need the following well known fact:

REMARK 7.2. If a domain is strongly pseudoconvex, then there exists a plurisubharmonic solution for the Dirichlet problem for continuous data on the boundary (see $[\mathrm{B}]$ and [Wa, Theorem 1]). In particular, for any point $z$ on the boundary, $\widehat{\mathcal{J}}_{z}=\left\{\delta_{z}\right\}$ holds for strongly pseudoconvex domains by Corollary 6.4.

EXAmple 7.3. Take a smooth function $\eta: \mathbb{R} \rightarrow \mathbb{R}$ with the following properties:

(1) $\eta(x) \geq 0, \eta$ is even and convex.

(2) $\eta^{-1}(0)=[-a, a]$, where $0<a<1$.

(3) There exists $b>0$ such that $\eta(x)>1$ when $|x|>b$.

(4) The map $u(z, w)=|z|^{2}+|z-1|^{2}|w|^{2}+\eta\left(|w|^{2}\right)$ defined on $\mathbb{C}^{2}$ has non-zero gradient on the level set $u(z, w)=1$.

We can satisfy condition (4) using Sard's theorem. Set

$$
D=\left\{(z, w):|z|^{2}+|z-1|^{2}|w|^{2}+\eta\left(|w|^{2}\right)<1\right\} .
$$

It is easy to calculate the determinant of the Levi form of $u$ :

$$
\mathcal{L} u=|z-1|^{2}+\left(1+|w|^{2}\right)\left[\eta^{\prime}\left(|w|^{2}\right)+|w|^{2} \eta^{\prime \prime}\left(|w|^{2}\right)\right] .
$$

Now $D$ is a bounded strongly hyperconvex domain with a smooth boundary. To get a star-shaped domain, we take $b \leq 1 / 64$. For $(z, w) \in D$, let $r=|z|$ and $s=|w|$; then $r \leq 1$ and $s \leq 1 / 8$. We need to show that $(t z, t w) \in D$ for all $t \in(0,1)$. We show that

$$
u(t z, t w)<u(z, w)
$$

for all $t \in(0,1)$. A direct calculation shows that this is true if

$$
2\left(1-t^{3}\right) s^{2} r<\left(1-t^{2}\right) r^{2}+\left(1-t^{4}\right) s^{2} r^{2}+\left(1-t^{2}\right) s^{2} .
$$

If $r \leq 1 / 4$, then

$$
2\left(1-t^{3}\right) s^{2} r<\left(1-t^{2}\right) s^{2}
$$

and if $1 / 4 \leq r \leq 1$, then

$$
2\left(1-t^{3}\right) s^{2} r<\frac{1}{16}\left(1-t^{2}\right) \leq\left(1-t^{2}\right) r^{2}+\left(1-t^{4}\right) s^{2} r^{2}+\left(1-t^{2}\right) s^{2} .
$$

In either case (7.1) is true, thus $D$ is star-shaped.

To show that $\partial D$ is not $c$-regular, we need to find a non- $c$-regular point on the boundary. Take $t_{j} \rightarrow \pi / 2$ and $r_{j} \neq 0$ so that

$$
r_{j}=\frac{-2 \cos t_{j}}{1+s_{j}^{2} \cos ^{2} t_{j}}
$$

where $s_{j} \rightarrow 0$. Set

$$
p_{j}=\left(1+r_{j} e^{i t_{j}}, s_{j}\right) \text {. }
$$


Then $p_{j} \in \partial D$ and $p_{j} \rightarrow p=(1,0) \in \partial D$. Evidently

$$
\mathcal{L} u\left(p_{j}\right)=r_{j}^{2} \downarrow \mathcal{L} u(p)=0 .
$$

For some open sets $V_{j} \subset \bar{D}$ with $p_{j} \in \partial V_{j}$ we have $\mathcal{L} u\left(V_{j}\right)>0$. Then $V_{j}$ are strongly pseudoconvex, hence by Remark $7.2, \mathcal{J}_{p_{j}}^{\mathrm{c}}=\left\{\delta_{p_{j}}\right\}$ for all $j$. To show that $\partial D$ is not $c$-regular at $p$, we need to find a Jensen measure $\mu \in \widehat{\mathcal{J}}_{p}$ such that $\mu \neq \delta_{p}$.

Consider the analytic disk $f: \bar{U} \rightarrow \bar{D}$ defined for $\zeta \in \bar{U}$ as

$$
f(\zeta)=(1, a \zeta)
$$

and consider the Jensen measure $\mu_{f}$ induced by $f$. Then $f$ is the uniform limit of the analytic maps $f_{j}: \bar{U} \rightarrow D$ defined by

$$
f_{j}(\zeta)=(1-1 / j, a \zeta)
$$

Therefore, $\mu_{f_{j}} \in \widehat{\mathcal{J}}_{v_{j}}$, where $v_{j}=(1-1 / j, 0) \in D$. Since $v_{j} \rightarrow p$, we see that $\mu_{f} \in \widehat{\mathcal{J}}_{p}$ and it is not equal to the Dirac measure. Take a continuous function $\varphi$ defined on $\bar{D}$ so that $\varphi\left(p_{j}\right)=1$ for all $j$, and $\varphi(1, a \zeta) \equiv 0$ on $\{|\zeta|=1\}$. Then $\widehat{E} \varphi\left(p_{j}\right)=\varphi\left(p_{j}\right)=1$ for all $j$ since $\widehat{\mathcal{J}}_{p_{j}}=\left\{\delta_{p_{j}}\right\}$ and

$$
\widehat{E} \varphi(p) \leq \int \varphi d \mu_{f}=0 \text {. }
$$

Thus $\widehat{E} \varphi$ is not continuous, therefore $\partial D$ is not $c$-regular. Note that by the same reasoning as in the proof of Theorem 5.9, if $f$ is the restriction of $\varphi$ to the boundary $\partial D$, then the envelope $\mathcal{S} f$ coincides with $E^{\mathrm{b}} \varphi$ on $\partial D$. Therefore the envelope $\mathcal{S} f$ is not continuous either.

REMARK. The set of strongly regular points is not closed in this example. Thus the assumption that the domain is $c$-regular is necessary in Corollary 6.5.

\section{References}

[B] H. J. Bremermann, On a generalized Dirichlet problem for plurisubharmonic functions and pseudoconvex domains. Characterization of Šilov boundaries, Trans. Amer. Math. Soc. 91 (1959), 246-276.

[BS] S. Q. Bu and W. Schachermayer, Approximation of Jensen measures by image measures under holomorphic functions and applications, ibid. 331 (1992), 585608.

[CCW] M. Carlehed, U. Cegrell and F. Wikström, Jensen measures, hyperconvexity and boundary behaviour of the pluricomplex Green function, Ann. Polon. Math. 71 (1999), 87-103.

[CR] B. J. Cole and T. J. Ransford, Jensen measures and harmonic measures, J. Reine Angew. Math. 541 (2001), 29-53.

[C] J. B. Conway, A Course in Functional Analysis, 2nd ed., Grad. Texts in Math. 96, Springer, 1990. 
[K] M. Klimek, Pluripotential Theory, London Math. Soc. Monogr. 6, Oxford Univ. Press, 1991.

[P1] E. A. Poletsky, Holomorphic currents, Indiana Univ. Math. J. 42 (1993), 85-144.

[P2] -, Plurisubharmonic functions as solutions of variational problems, in: Several Complex Variables and Complex Geometry (Santa Cruz, CA, 1989), Part 1, Proc. Sympos. Pure Math. 52, Part 1, Amer. Math. Soc., 1991, 163-171.

[P3] - Jensen measures and analytic multifunctions, Ark. Mat. 42 (2004), 335-352.

[R] T. J. Ransford, Jensen measures, in: Approximation, Complex Analysis, and Potential Theory (Montréal, QC, 2000), NATO Sci. Ser. II Math. Phys. Chem. 37, Kluwer, 2001, 221-237.

[S] N. Sibony, Une classe de domaines pseudoconvexes, Duke Math. J. 55 (1987), 299-319.

[Wa] J. B. Walsh, Continuity of envelopes of plurisubharmonic functions, J. Math. Mech. 18 (1968), 143-148.

[W] F. Wikström, Jensen measures and boundary values of plurisubharmonic functions, Ark. Mat. 39 (2001), 181-200.

Department of Mathematics, 215 Carnegie Hall

Syracuse University

Syracuse, NY 13244, U.S.A.

E-mail: nggogus@syr.edu

Reçu par la Rédaction le 14.2.2005

Révisé le 18.9.2005 